\title{
Traduire
}

Revue française de la traduction

$237 \mid 2017$

La tête dans la toile

\section{Parlons du localisateur : rôle et profil}

\section{Ma'moun Alshtaiwi}

\section{(2) OpenEdition \\ Journals}

Édition électronique

URL : http://journals.openedition.org/traduire/945

DOI : 10.4000/traduire.945

ISSN : 2272-9992

Éditeur

Société française des traducteurs

Édition imprimée

Date de publication : 1 décembre 2017

Pagination : 38-47

ISSN : 0395-773X

\section{Référence électronique}

Ma'moun Alshtaiwi, « Parlons du localisateur : rôle et profil », Traduire [En ligne], 237 | 2017, mis en

ligne le 01 décembre 2017, consulté le 29 juin 2019. URL : http://journals.openedition.org/traduire/945 ; DOI : 10.4000/traduire.945 


\title{
Parlons du localisateur : rôle et profil
}

\author{
Ma'moun Alshtaiwi
}

\section{Introduction}

L'évolution des Technologies de l'Information et de la Communication (TIC) a introduit une nouvelle dimension dans les activités traditionnelles du traducteur, tant en ce qui concerne les outils de travail que son rôle dans la communication interlinguistique. Actuellement, l'activité du traducteur fait souvent appel à différentes compétences en informatique, aussi bien pour des tâches de base comme le traitement de texte que pour la documentation à partir d'ouvrages au format numérique, de ressources terminologiques disponibles sur internet (dictionnaires électroniques, glossaires techniques), de recherche d'informations y compris la fréquence des occurrences, voire la gestion de forums de discussion en ligne et de listes de diffusion.

Depuis quelques années, les produits et processus issus des nouvelles technologies sont devenus l'objet même de l'activité de traduction car les développeurs ont rapidement constaté le besoin de les rendre accessibles à un public international. Ainsi, le traducteur joue un rôle important dans la mondialisation des produits informatiques, qui exige une adaptation, voire une redéfinition, des composantes de son métier. De cette interpénétration entre traduction et mondialisation découle la nécessité de préciser les caractéristiques essentielles du profil du traducteur spécialisé en localisation de produits informatiques.

L'objectif de cet article est donc d'explorer cet ensemble de caractéristiques ainsi que d'autres aspects liés au domaine de la localisation. Quelques considérations évoquées dans les travaux référencés feront l'objet d'une synthèse suivie de réflexions personnelles, en vue de présenter une vision plus holistique de la problématique.

\section{Définitions : localisation, mondialisation et internationalisation}

L'étape de la localisation est précédée de l'internationalisation, qui consiste à réélaborer les produits afin de faciliter leur introduction et leur adaptation à l'international. La localisation est une des étapes de la mondialisation qui consiste à adapter un produit et tout ce qui l'environne 
(processus, concepts, documentation...) aux normes culturelles et langagières ainsi qu'aux attentes du marché-cible (Döhler, 1997).

Le domaine de la localisation peut être défini comme l'ensemble des opérations de traduction et d'adaptation d'un contenu numérique pour un environnement cible. Elle concerne notamment l'adaptation dans une autre langue et dans telle ou telle culture de produits informatiques, de pages internet, de sites web et de guides d'utilisation. La localisation repose donc également sur des compétences techniques et informatiques avancées.

La mondialisation, quant à elle, désigne les liens d'interdépendance entre les hommes et leurs activités à l'échelle mondiale. Ce terme est utilisé pour désigner la diffusion mondiale des informations et l'expansion des activités économiques.

L'internationalisation doit avoir lieu au moment de la conception du produit afin d'éviter des modifications postérieures, qui entraîneraient un surcoût financier. II est évident que la composante linguistique doit être prise en compte dans l'ensemble de ce processus, à savoir au niveau des produits eux-mêmes mais aussi au niveau de leur diffusion et de leur exploitation (Gouadec, 2003). Pour internationaliser un produit informatique ou multimédia, au moins trois acteurs devront intervenir : le concepteur (chargé d'internationaliser les produits), le rédacteur (chargé d'internationaliser les documents qui accompagnent les produits) et le traducteur (chargé de traduire et de localiser la composante linguistique). D'où notre intérêt pour la définition du rôle du traducteur-localisateur dans le cycle de la mondialisation, ainsi que de ses besoins pour faire face aux nouveaux marchés dans les secteurs concernés.

\section{Rôle du traducteur dans l'internationalisation}

Si l'internationalisation consiste à rendre utilisable un produit partout dans le monde, le traducteur doit, quant à lui, prendre en compte toutes les résistances éventuelles à cette démarche. Autrement dit, il doit réfléchir aux modes de conceptualisation, aux raisonnements, aux systèmes de valeurs, aux points de vue et même aux éventuels préjugés propres aux pays où sera commercialisé le produit.

À cet égard, l'intervention du traducteur, que nous appellerons dorénavant "localisateur ", s'organise en plusieurs séquences. Tout d'abord en amont de la fabrication du produit, puisqu'il est celui qui maîtrise les spécificités langagières, anticipe les écarts prévisibles et peut signaler les éléments linguistiques déjà existants. II livre ensuite le résultat de sa prestation de traduction. Enfin, il intervient en aval de la traduction pour la révision et la vérification de l'adéquation du produit aux représentations culturelles et aux pratiques langagières du public-cible.

D'après les travaux de Daniel Gouadec (2003 : 529), l'internationalisation d'un produit s'effectue en cinq étapes, au cours desquelles le localisateur intervient à divers titres. La pre- 
mière phase correspond au " développement logiciel ", étant donné que la localisation modifie le produit en lui-même. La phase suivante est le " traitement informatique du matériau à traduire ", c'est-à-dire, le traitement du code, suivi du " retraitement " de certaines composantes du produit, par exemple l'interface graphique, à laquelle succède la " traduction-adaptation " des matériaux linguistiques composant ce produit. À la fin du processus, il ne reste qu'une phase de traduction, celle des documents d'accompagnement (fichiers d'aide, fichier lisezmoi, guides utilisateur).

La description qui précède peut se représenter de la manière suivante :

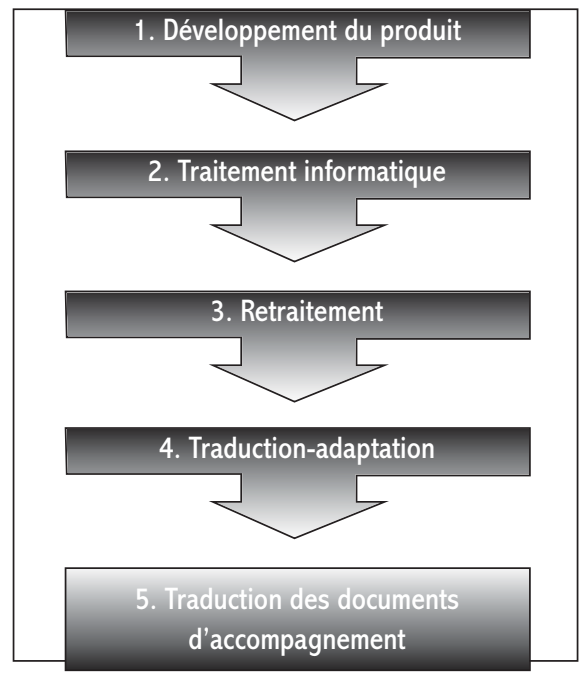

Phases de l'internationalisation d'un produit (logiciel ou site Web)

L'auteur souligne que ce processus s'adapte à la pratique de la localisation dans les deux marchés principaux : la localisation de sites Web et la localisation de logiciels. II va de soi que la première moitié du schéma concerne principalement les concepteurs - les informaticiens tandis que la seconde concerne les localisateurs. Ces derniers peuvent ainsi être amenés à travailler sur de nombreux types de documents et de formats de fichiers numériques et, par conséquent, doivent avoir des connaissances pointues dans le domaine des TIC. Ce domaine de compétences représente l'enjeu principal dans la définition du profil du localisateur, comme nous le verrons dans la section suivante.

\section{Profil du localisateur}

Le localisateur joue un rôle essentiel et majeur dans ce processus puisqu'il est chargé d'adapter non seulement des éléments linguistiques mais aussi de nombreux aspects culturels (picto- 
grammes, image, sons, etc.). Son profil ne se définit pas seulement par son savoir-faire en matière de traduction mais également par sa connaissance approfondie du traitement des produits à localiser.

Le localisateur doit avoir une bonne maîtrise de la traduction spécialisée, de la réécriture et de la gestion de projets. II doit être capable, grâce à sa connaissance de l'informatique et des outils adéquats, de traiter le matériau à localiser, si nécessaire en modifiant les menus, les icônes, les boîtes de dialogues, etc.

Pour mener à bien son travail, il doit savoir intervenir au-delà de l'opération de traduction pure, car, quel que soit le matériau qu'il aura à traiter, le localisateur devra accomplir certaines tâches qui nécessiteront la maîtrise des aspects théoriques et pratiques de l'internationalisation. Grâce à la mise en œuvre de ces diverses compétences, il sera en mesure de mettre en place une véritable « méthode de la localisation : vérification de l'internationalisation, établissement de la terminologie, pseudo-traduction, traduction, contrôle de la qualité, etc. " (Quirion, 2003 : 550). Ainsi, le profil du localisateur rassemble une série de compétences que nous pouvons classer en deux groupes : les anciennes et les nouvelles.

\subsection{Compétences anciennes}

Traducteur spécialisé, le localisateur réunit, selon Gouadec (2003 : 534), les caractéristiques du traducteur "le plus abouti ", car il est supposé disposer de connaissances en phraséologie, en rédaction, en relecture, en révision, en adaptation et en réécriture, afin d'être capable de traduire tout type de texte. II s'agit donc de l'ensemble de compétences qui caractérisent le "bon traducteur ", appliquées à des domaines spécialisés très divers.

L'exercice de la localisation, comme toute activité de traduction, se fonde également sur l'activité terminologique. À la différence de Gouadec, Quirion (2003) insiste particulièrement sur l'importance de la pratique terminologique dans la localisation.

La terminologie constitue donc la pierre angulaire des activités traductionnelles, qu'elles soient bilingues ou multilingues, dans la pratique de la localisation. En effet, les localisateurs sont amenés à établir des glossaires terminologiques qui constitueront un guide (individuel ou collectif) pour la définition des équivalents dans les langues cibles, ainsi qu'un outil pour assurer la cohérence linguistique du produit à localiser. La terminologie introduit ainsi sa démarche onomasiologique au travers du produit à localiser, parce qu'adapter culturellement implique d'aller au-delà du terme pour se pencher sur le ou les concepts.

Selon Quirion (2003 : 548), deux axes sous-tendent cette recherche terminologique : l'axe horizontal qui consiste en une recherche terminologique dans le domaine concerné (par exemple une recherche terminologique dans les références juridiques de la culture cible) et l'axe vertical qui tient compte de la famille du produit afin de veiller à ce qu'une cohérence rigoureuse 
s'opère entre les différentes versions de celui-ci. Dans le cas d'un logiciel, on respectera ainsi la terminologie adoptée lors de la précédente édition.

\subsection{Compétences nouvelles}

Les besoins actuels du marché et les progrès rapides de la technologie contraignent les localisateurs à développer de nouvelles compétences au fur et à mesure de l'évolution du secteur.

Une des compétences souhaitées par les professionnels, et qui n'est pourtant pas mise en relief par la définition du métier de localisateur proposée par la LISA (Localisation Industry Standard Association), est la spécialisation dans les composants informatiques et multimédias. À partir de cet ensemble de compétences, le profil idéal du localisateur correspondrait à ce que Gouadec (2003) appelle "un ingénieur en communication multilingue multimédia ". Nous précisons qu'il ne s'agit pas d'un nouveau profil de développeur informatique, mais bien d'un traducteur spécialisé, dont les compétences doivent s'adapter à l'ensemble de ses nouvelles activités comme le dit bien Gouadec (2003 : 537) :

au terme de l'analyse de son profil de compétences, le localiseur/localisateur apparaît comme un excellent traducteur spécialisé (multispécialisé) maîtrisant parfaitement tous les métiers de la traduction ou métiers contribuant à la prestation du traducteur (documentation, terminographie, phraséographie, rédaction, relecture, révision, adaptation, réécriture, gestion, achatvente...), maîtrisant les outils standard et trouvant, dans l'informatique et par l'informatique, des marchés à très fortes plus-values.

\section{Place du traducteur dans le marché de la localisation}

Une fois définies les compétences et les qualités que doit réunir le traducteur-localisateur, il nous semble nécessaire de donner un bref aperçu de la place qu'il occupe dans le marché de la localisation. À cet égard, Döhler (1997) affirme que le traducteur doit non seulement maîtriser les outils informatiques inhérents à la localisation, mais aussi avoir une vaste connaissance du marché et des produits à localiser, ainsi qu'identifier sa place dans le cycle, compte tenu de ses propres limitations.

II s'agit donc de définir les prestations de base et les prestations supplémentaires inhérentes à un spécialiste en informatique et qui vont constituer la valeur ajoutée au travail du traducteur. Ce nouveau marché génère des situations concurrentielles auxquelles doit faire face le traducteur indépendant, car les projets de localisation exigent souvent une grande gamme de services, de l'expérience technique et des prestations rapides et efficaces, caractéristiques qui sont autant de prérogatives des groupes ou sociétés de localisation. 


\subsection{Services informatiques versus Services langagiers}

Compte tenu de l'ampleur des projets de localisation, les localisateurs indépendants choisissent souvent de se rapprocher d'une société qui jouera le rôle de médiatrice entre le client et le localisateur. Gouadec (2003 : 538) fait référence à la rivalité existante, en Europe, entre deux groupes principaux de sociétés :

- Les sociétés de service informatique, consacrées aux applications techniques et qui assument également les tâches de traduction (en sous-traitant auprès d'un traducteur indépendant, en ayant recours à un technicien qui maîtrise un peu la langue d'arrivée, voire à un logiciel de traduction).

- Les sociétés de services langagiers ou d'ingénierie linguistique, qui assurent des prestations linguistiques aussi bien qu'informatiques, qui comptent sur des traducteurs ayant de solides connaissances en informatique (salariés ou indépendants) et éventuellement sur des informaticiens en sous-traitance.

Dans le contexte de la mondialisation, il paraît évident que les localisateurs doivent s'organiser en équipes de travail capables d'assurer des prestations à la hauteur des exigences du marché. Il paraît donc fondamental que les localisateurs, qui veilleront à maintenir à jour leurs compétences techniques, revendiquent leur place sur ces marchés très compétitifs, tout en mettant en valeur l'importance de la qualité linguistique qui reste souvent subordonnée aux prestations techniques.

\section{Choix du traducteur}

Le marché de la traduction, en mutation constante, notamment en raison de l'importance croissante de la composante informatique, contraint le traducteur à renouveler en permanence sa stratégie pour faire face aux nouvelles exigences des clients et aux nouvelles dimensions du métier de localisateur.

Comme l'indique Gouadec (2003 : 538), le traducteur a toujours le choix de n'accomplir que les tâches " dont il a l'habitude ", c'est-à-dire les tâches inhérentes à la traduction d'un texte écrit ou oral (interprétation, réexpression, relecture, adaptation si nécessaire, etc.). Cette stratégie serait la moins rentable car le client serait obligé de demander l'intervention d'un spécialiste en informatique capable d'achever le processus de localisation. Par conséquent, la solution la plus pertinente pour l'entreprise serait de contacter directement la concurrence (des techniciens qui " font " de la traduction, des sociétés de courtage et des sociétés de services linguistiques et langagiers).

À l'inverse, le traducteur peut choisir de conquérir ces nouveaux marchés, en mettant en avant la valeur ajoutée apportée à son travail par la composante technologique et la possibilité de 
se former pour assumer cet aspect inhérent de la localisation. Nous considérons que cette approche lui serait la plus favorable, même si elle implique des enjeux importants en termes d'investissements en apprentissage.

\section{Formation du localisateur}

Après avoir esquissé les principales caractéristiques du profil du localisateur, il convient à présent d'envisager les moyens d'acquisition des compétences tant linguistiques que technologiques nécessaires. Pour les deux niveaux de compétences requises, Quirion (2003) examine les deux programmes universitaires possibles pour l'enseignement de la localisation : les programmes spécialisés en localisation destinés aux diplômés en informatique et ceux qui s'adressent aux diplômés en traduction. S'ajoute une troisième option de formation visant les candidats n'ayant aucune expérience, ni en traduction ni en technologie.

Le premier programme impliquera des enjeux de formation linguistique énormes, dont la maîtrise d'au moins deux langues pour être initié à la traduction. La deuxième option, à l'attention des traducteurs souhaitant se spécialiser dans les outils et les méthodes informatiques est le type de programme le plus fréquemment proposé par les universités, même si seuls quelques éléments isolés du métier de la traduction (la traduction assistée par ordinateur, la conception de sites web, etc.) sont proposés. Le troisième programme impliquera une formation parallèle et approfondie dans les deux composantes ; linguistique et technologique. Cette option semble encore moins envisageable que la première. Il existe pourtant des formations de courte durée proposées aux non-spécialistes, ainsi que des cours ad hoc (notamment dans le domaine de la technologie et de la gestion de projet).

Quirion souligne ainsi l'importance de définir des programmes pour la formation de localisateurs qui soient axés sur l'acquisition des compétences nécessaires pour affronter les nouveaux marchés. Les options en formation continue s'avèrent plus nombreuses : elles sont dispensées par des universités, des associations de traducteurs ou des organisations privées, mais d'aucuns ne voient là qu'une solution provisoire et limitée.

Pym (2013) propose d'enseigner les compétences techniques fondamentales et de préparer les diplômés à avoir une vision critique d'ensemble. II invite en effet à former les diplômés en compétences techniques fondamentales de manière à protéger l'industrie de la localisation et, ce qui lui semble plus important encore, à inciter ces étudiants à avoir une vision critique de l'ensemble, à comprendre comment la technologie peut influencer la communication entre les cultures et à quel point les pratiques actuelles dans le secteur de la localisation privilégient les chiffres au détriment de la communication.

Après une recherche rapide sur les offres de formation en traduction et en localisation en France, force est de constater qu'il y a de plus en plus de programmes universitaires axés sur 
ce métier requérrant des compétences multiples. Même si l'équilibre entre les composantes linguistiques et technologiques n'est pas encore évident, on pourrait se réjouir du fait que la nécessité d'une certaine expertise préalable, au moins académique, en traduction semble désormais attendue.

De même, Gouadec (2003) réaffirme ce besoin actuel de proposer des formations complémentaires aux traducteurs déjà formés, dont le programme serait consacré à l'analyse des produits ou matériaux à localiser, à la mise au point des techniques de traduction de ces matériaux et à l'acquisition des compétences technologiques inhérentes à ce processus.

Une expérience de l'enseignement de la traduction permet d'affirmer que la familiarisation avec les outils informatiques est indispensable dans toute formation à la localisation, mais la base fondamentale de ce domaine sera toujours la traduction, l'informatique n'étant " qu'un outil au service du traducteur " (Gouadec, 2003 : 542). Ainsi, les compétences requises dans la localisation de produits et supports informatiques constituent-elles non seulement la base de ce nouveau profil du traducteur, mais aussi le point de départ pour la reformulation des programmes universitaires existants.

\section{Conclusion}

La redéfinition des compétences du traducteur s'avère nécessaire pour l'adapter aux nouveaux marchés du travail, qui eux-mêmes s'inscrivent dans la mondialisation des produits à contenu numérique. Comme on vient de le voir, bien qu'il s'agisse d'un nouveau métier, avec des caractéristiques de plus en plus spécifiques, la localisation est avant tout une variante de la traduction, spécialisée dans le traitement de matériaux informatiques et multimédias. D'où la dénomination proposée par Gouadec $(2003: 541)$ pour mieux cerner ce profil à compétences multiples : "ingénieur en communication multilingue multimédia ".

À l'ensemble des compétences anciennes et nouvelles s'ajoute le besoin de revendiquer la place du traducteur dans ces nouveaux marchés extrêmement compétitifs. L'enjeu est alors double, car il ne s'agit pas seulement de conquérir " la part du gâteau " qui reste souvent reléguée à des sociétés de services techniques (qui elles-mêmes sous-traitent aux traducteurs, si besoin est), il faut aussi trouver des stratégies pour s'adapter aux nouvelles exigences des clients et aux nouveaux produits à traiter.

En conséquence de quoi, la formation à la localisation s'avère essentielle pour un traducteur qui souhaite pénétrer ces marchés émergents. La formation continue reste certes l'option la plus rapide pour assurer cette formation complémentaire en technologies, néanmoins, force est de constater que dans bon nombre d'universités, les programmes de formation initiale en traduction introduisent depuis plusieurs années des enseignements davantage axés sur les métiers de la localisation. Pour autant, les problèmes liés notamment à la formation de 
formateurs en localisation et à l'acquisition des ressources matérielles nécessaires à cette formation perdurent.

Les recherches menées dans ce domaine spécialisé de la traduction nous permettent d'observer que la plupart des études sur la localisation suivent une approche purement technique ou économique. De même que très peu de spécialistes en localisation se consacrent à l'enseignement, très peu de chercheurs s'investissent dans des études sur les divers aspects de la localisation en tant qu'activité de traduction, ce qui semble étonnant au regard des enjeux académiques et économiques concernés. La revendication devrait probablement commencer par la normalisation du terme le plus approprié pour ce nouveau profil de traducteur, appelé indistinctement localisateur, localiseur, traducteur-localisateur, traducteur spécialiste en localisation et ingénieur en communication multilingue multimédia.

mamoun.alshtaiwi@gmail.com

Ma'moun Alshtaiwi est docteur en Lexicologie, Terminologie Multilingues, Traduction. Sa thèse intitulée "Structuration ontologique et étude sémantico-syntaxique de la terminologie des sciences et techniques spatiales (arabe-français-anglais) " a été soutenue en 2016 à l'université Lumière Lyon 2.

\section{Bibliographie}

CHUKWU Uzoma, 1996, "La fonction traduction en localisation logicielle ", in ASP, Actes du 17e colloque du GERAS, p. 135-146.

DÖHLER Per N, 1997, "Facets of Software Localization A Translator's View ", Translation Journal 1, Volume 1, http://accurapid.com/journal/softloc.htm.

ESSELINK Bert, 2000, A Practical Guide to Localization, Amsterdam et Philadelphia (ÉtatsUnis), Benjamins.

GAMBIER Yves, 2006, "Mondialisation en cours et traduction » in Meta, vol. 51, № 4. Montréal (Canada) : Presses de l'Université de Montréal. 848-853.

GOUADEC Daniel, 2002, Profession : traducteur, Paris, Maison du Dictionnaire, p. 51-70.

GOUADEC Daniel, 2003, "Le bagage spécifique du localiseur/localisateur, le vrai " nouveau profil " requis ", http:www.erudit.org/revue/meta/2003/v48/n4/008724ar.html: Les Presses de l'Université de Montréal, Canada, p. 526-545. 
QUIRION Jean, 2003, "La formation en localisation à l'université : pour quoi faire ? ", http:www.erudit.org/revue/meta/2003/v48/n4/008724ar.html, Les Presses de l'Université de Montréal (Canada), p. 546-558.

QUIRION Jean, 2006, "La localisation, palimpseste de l'aménagement terminologique? Stratégies d'implantation terminologique et marketing ", in Meta, vol. 51, N 4, Presses de I'Université de Montréal (Canada), p. 824-837.

PYM Anthony, 2014, "Localization, Training and Instrumentalization ", Tarragona (Espagne), in Intercultural Studies Group, Universitat Rovira i Virgili, Translation Research Projects, ed. Esther Torres Simon et David Orrego Carmona, p. 37-50. 